\title{
Konstantinov effect in helium II
}

\author{
L.A. Melnikovsky \\ P.L. Kapitza Institute for Physical Problems, Moscow, Russia \\ E-mail: leva@kapitza.ras.ru
}

Received November 22, 2007

\begin{abstract}
We consider reflection of first and second sound waves by a rigid flat wall in helium II. A nontrivial dependence of the reflection coefficients on the angle of incidence is obtained. Sound conversion is predicted at slanted incidence.
\end{abstract}

PACS: 67.25.dg Transport, hydrodynamics, and superflow; 67.25.dt Sound and excitations; 67.55.dm Two-fluid model; phenomenology.

Keywords: second sound, reflection coefficients, sound absorption, strong anisotropy.

\section{Introduction}

Sound absorption in air at a plane surface may be surprisingly high and has strong anisotropy $[1,2]$. This phenomenon is very important in acoustics and is sometimes referred to as Konstantinov effect. Qualitative explanation of the effect is two fold [3].

- Air velocity in an oblique wave has a non-zero component tangential to the surface. At the surface itself, however, the velocity is clamped. Large velocity gradient in a thin boundary layer results is a large viscous dissipation of energy.

- Not only the velocity, but also the temperature, undergo periodic oscillation in a sound wave. Temperature of the wall (and adjoined gas), however, is constant. Thermal conductance in presence of a large temperature gradient in the boundary layer again leads to high energy dissipation.

Helium II supports two types of motion and its hydrodynamics is much richer than that of a gas. The former features two independent sound modes: first sound and second sound [4]. Due to anomalously small thermal expansion of helium, these modes can be viewed as purely pressure and temperature waves, respectively. To solve the problem of sound reflection in superfluid one must take both modes into account.
Propagation of multiple bulk modes in helium II is a consequence of existence of additional hydrodynamic variables. In Sec. 2 we find all three nontrivial harmonic (i.e., proportional to $\exp (i \mathbf{k r}-i \omega t))$ solutions of linear superfluid hydrodynamic equations.

Some of the variables are effectively eliminated in restricted geometry. In particular, a steady cell wall eliminates the perpendicular component of the mass flux $\mathbf{j}$ and the normal velocity $\mathbf{v}_{n}$ : the boundary condition* at the wall is

$$
\mathbf{j}_{\perp}=0, \quad \mathbf{v}_{n}=0 .
$$

Fourth sound [5] is one result of such elimination. This is the only sound mode in a narrow channel, both temperature and pressure oscillating coherently in this wave. In general, the sound modes, independent in bulk liquid, begin to interact at the boundary**. The boundary condition (1) selects a two-dimensional subspace of solutions for particular frequency $\omega$. Specific solutions correspond to the reflection of first and second sound (Secs. 3 and 4). Interestingly enough, incident sound energy is not only reflected and dissipated as it happens in a normal fluid, but is also converted between first and second sound.

* The heat transfer through the interface at low temperature can be neglected due to Kapitza resistance. The heat flux at the interface vanishes simultaneously with the perpendicular component of the normal velocity $\mathbf{v}_{n \perp}$.

** Sound reflection at the free helium surface and at the solid helium boundary is extensively explored [6-9]. 


\section{Harmonic solutions}

Consider the linearized equations of superfluid hydrodynamics [10]:

$$
\begin{gathered}
\dot{\rho}+\frac{\partial j^{i}}{\partial x^{i}}=0 \\
\dot{j}^{i}+\frac{\partial p}{\partial x^{i}}=\eta \frac{\partial}{\partial x^{k}}\left(\frac{\partial v_{n}^{i}}{\partial x^{k}}+\frac{\partial v_{n}^{k}}{\partial x^{i}}-\delta^{i k} \frac{2}{3} \frac{\partial v_{n}^{l}}{\partial x^{l}}\right)+ \\
+\frac{\partial}{\partial x^{i}}\left(\zeta_{2} \frac{\partial v_{n}^{l}}{\partial x^{l}}-\zeta_{1} \frac{\partial \rho_{s} w^{l}}{\partial x^{l}}\right), \\
v_{s}^{k}+\frac{\partial \mu}{\partial x^{k}}=\frac{\partial}{\partial x^{k}}\left(\zeta_{4} \frac{\partial v_{n}^{l}}{\partial x^{l}}-\zeta_{3} \frac{\partial \rho_{s} w^{l}}{\partial x^{l}}\right), \\
T\left(\dot{\sigma} \rho+\sigma \dot{\rho}+\sigma \rho \frac{\partial v_{n}^{l}}{\partial x^{l}}\right)=\kappa \frac{\partial^{2} T}{\partial x^{l}},
\end{gathered}
$$

where $\eta, \zeta_{1}=\zeta_{4}, \zeta_{2}, \zeta_{3}, \kappa$ are dissipative coefficients, $\sigma$ is entropy per unit mass, $p, \mu, T$ are pressure, chemical potential, and temperature, $\mathbf{v}_{s}, \mathbf{v}_{n}$, and $\mathbf{w}=\mathbf{v}_{n}-\mathbf{v}_{s}$ are superfluid, normal, and relative velocities, and $\rho$ and $\mathbf{j}$ are mass and momentum densities. The velocities and the momentum density are coupled by the equation

$$
\mathbf{j}=\rho \mathbf{v}_{s}+\rho_{n} \mathbf{w}=\rho \mathbf{v}_{n}-\rho_{s} \mathbf{w} .
$$

Further simplification is facilitated by ignoring thermal expansion (we therefore disregard the difference between specific heats $c=T \partial \sigma / \partial T$ at constant pressure and at constant volume). Namely put

$$
\begin{gathered}
p^{\prime}=s^{2} \rho^{\prime}, \\
\rho \mu^{\prime}=-\sigma \rho T^{\prime}+p^{\prime}=-\sigma \rho T^{\prime}+s^{2} \rho^{\prime}, \\
T \sigma^{\prime}=c T^{\prime},
\end{gathered}
$$

where $s=(\partial p / \partial \rho)^{1 / 2}$ is the first sound velocity. The prime denotes small deviation of the variables from their equilibrium values.

In a harmonic perturbation, the space and time dependence of all deviations takes the form*

$$
\exp (i \mathbf{k r}-i \omega t)
$$

To find all possible harmonic excitations in bulk helium we substitute this exponential term in Eqs. (2)-(5) and keep linear terms only.

Mass conservation (2) gives

$$
\omega \rho^{\prime}=k^{i} j^{i} .
$$

The momentum conservation law (3) can be transformed as follows:

$$
\begin{aligned}
&-i \omega j^{i}+i p^{\prime} k^{i}=-\eta k^{k}\left(v n^{i} k^{k}+k^{i} v_{n}^{k}-\frac{2}{3} \delta^{i k} k^{l} v_{n}^{l}\right)- \\
&-k^{i} k^{k}\left(-\rho_{s} \zeta_{1} w^{k}+\zeta_{2} v_{n}^{k}\right),
\end{aligned}
$$

and, using (7), (6), and (11)

$$
\begin{aligned}
\left(\omega+i \eta k^{2} / \rho\right) j^{i}+ & \left(i A-s^{2} / \omega\right) k^{i} k^{k} j^{k}+i \eta k^{2} \rho_{s} w^{i} / \rho+ \\
+ & +i B k^{i} k^{k} w^{k}=0,
\end{aligned}
$$

where the constants $A=\left(\eta / 3+\zeta_{2}\right) / \rho$ and $B=\left(A-\zeta_{1}\right) \rho_{s}$.

From the energy conservation law (5) for harmonic deviation we get

$$
T \omega \sigma^{\prime} \rho+T \omega \sigma \rho^{\prime}-T \sigma \rho v_{n}^{l} k^{l}+i k^{2} \kappa T^{\prime}=0 .
$$

Using (6), (9), and (11) this can be reduced to

$$
T \sigma \rho_{s} k^{i} w^{i}=\left(c \omega \rho+i \kappa k^{2}\right) T^{\prime} .
$$

Finally, substituting the exponential term (10) and (6) in (4) we obtain

$$
\begin{gathered}
-i \omega\left(j^{i}-\rho_{n} w^{i}\right) / \rho+i k^{i} \mu^{\prime}= \\
=-k^{i}\left(-\rho_{s} \zeta_{3} w^{k} k^{k}+\zeta_{4}\left(j^{k}+\rho_{s} w^{k)} k^{k} / \rho\right)\right.
\end{gathered}
$$

Combining this with (8), (11), and (13)

$$
\omega j^{i}+\left(i \zeta_{4}-s^{2} / \omega\right) k^{i} k^{k} j^{k}-\omega \rho_{n} w^{i}+G k^{i} k^{k} w^{k}=0,
$$

where

$$
\begin{gathered}
G=\rho_{s}\left[\frac{T \rho \sigma^{2}}{c \omega \rho+i \kappa k^{2}}+i\left(\zeta_{4}-\rho \zeta_{3}\right)\right] \approx \\
\approx \frac{T \rho_{s} \sigma^{2}}{c \omega}-i \rho_{s}\left[\frac{T \kappa k^{2} \sigma^{2}}{c^{2} \omega^{2} \rho}-\left(\zeta_{4}-\rho \zeta_{3}\right)\right] .
\end{gathered}
$$

Equations (12) and (14) can be written together as

$$
\hat{L}\left(\frac{j}{\mathbf{w}}\right)=0,
$$

where $\hat{L}$ is a square $6 \times 6$ matrix composed of the coefficients from (12) and (14). The linear system is consistent if $\operatorname{det} \hat{L}=0$. Due to the system isotropy, the determinant cannot depend on individual components of $k^{i}$. Instead it depends on $k^{2}=k^{i} k^{i}$ only. We therefore can put $k^{y}=k^{z}=0$ and treat $\hat{L}$ as a $4 \times 4$ matrix:

* It is important to mention that unbounded solutions (those with complex wave vector $\mathbf{k}$ ) should not be overlooked in a restricted geometry. 


$$
\hat{L}=\left(\begin{array}{cccc}
\omega+\left(-s^{2} / \omega+i A+i \eta / \rho\right) k^{2} & 0 & i\left(\eta \rho_{s} / \rho+B\right) k^{2} & 0 \\
0 & \omega+i \eta k^{2} / \rho & 0 & i \eta k^{2} \rho_{s} / \rho \\
\omega+\left(-s^{2} / \omega+i \zeta_{4}\right) k^{2} & 0 & -\omega \rho_{n}+G k^{2} & 0 \\
0 & \omega & 0 & -\omega \rho_{n}
\end{array}\right)
$$

After factorization $\operatorname{det} \hat{L}$ simplifies to

$$
\begin{gathered}
\operatorname{det} \hat{L}=\left|\begin{array}{cc}
\omega+\left(i A+i \eta / \rho-s^{2} / \omega\right) k^{2} & i\left(\eta \rho_{s} / \rho+B\right) k^{2} \\
\omega+\left(i \zeta_{4}-s^{2} / \omega\right) k^{2} & -\omega \rho_{n}+G k^{2}
\end{array}\right| \times \\
\times\left|\begin{array}{cc}
\omega+i \eta k^{2} / \rho & i \eta k^{2} \rho_{s} / \rho \\
\omega & -\omega \rho_{n}
\end{array}\right|
\end{gathered}
$$

$$
\begin{gathered}
\left(\begin{array}{c}
j_{I 1} \sin \phi_{1} \\
-j_{I 1} \cos \phi_{1} \\
0 \\
0
\end{array}\right)+\left(\begin{array}{c}
j_{R 1} \sin \phi_{1} \\
j_{R 1} \cos \phi_{1} \\
0 \\
0
\end{array}\right)+\left(\begin{array}{c}
0 \\
0 \\
w_{R 2} \sin \phi_{2} \\
w_{R 2} \cos \phi_{2}
\end{array}\right)+\left(\begin{array}{c}
\rho_{n} w_{3}^{x} \\
\rho_{n} w_{3}^{y} \\
w_{3}^{x} \\
w_{3}^{y}
\end{array}\right)= \\
=\left(\begin{array}{c}
-\rho_{s} w \\
0 \\
w \\
0
\end{array}\right)
\end{gathered}
$$

All nontrivial solutions immediately follow:

$$
\begin{gathered}
\omega^{2} \approx k_{1}^{2}\left(s^{2}-i \omega A-i \frac{\omega \eta}{\rho}\right) \approx \\
\approx k_{1}^{2}\left[s^{2}-i \frac{\omega}{\rho}\left(\frac{4 \eta}{3}+\zeta_{2}\right)\right] \approx k_{1}^{2} s^{2}, \\
\omega^{2} \approx k_{2}^{2} \omega\left(G-i \frac{\eta \rho_{s}}{\rho}-i B\right) / \rho_{n} \approx k_{2}^{2} \frac{T \rho_{s} \sigma^{2}}{c \rho_{n}} \equiv k_{2}^{2} s_{2}^{2}, \\
\omega=-i \eta k_{3}^{2} / \rho_{n},
\end{gathered}
$$

where $s_{2}$ is the second sound velocity.

Roots $\mathbf{k}_{1}$ (15) and $\mathbf{k}_{2}$ (16) correspond to «longitudinal» solutions where $j^{i} \propto w^{i} \propto k_{1,2}^{i}$, while the root $\mathbf{k}_{3}$ (17) corresponds to a «transverse» one $j^{i} k_{3}^{i}=w^{i} k_{3}^{i}=0$. The approximation in (15) and (16) is based on an assumption of low bulk damping, i.e. $\left|k_{3}\right|>>\left|k_{2}\right|>\left|k_{1}\right|$. This implies complete splitting between first and second sound, namely $\mathbf{w}=0$ for (15) and $\mathbf{j}=0$ for (16). In the third solution (17), the superfluid velocity vanishes $\mathbf{v}_{s}=0$, i.e. the mass flux and the relative velocity are coupled by the relation $\mathbf{j}=\rho_{n} \mathbf{w}$.

\section{First sound reflection}

To avoid complications associated with wall deformation, consider a perfectly rigid flat surface. We therefore ignore numerous peculiarities of sound transmission into solids. By $\phi_{1}$ denote the angle of incidence for the first sound wave (see Fig. 1). The subscripts $I 1, R 1$, and $R 2$ refer to the incident first, reflected first, and reflected second sound waves, respectively. The $x$ axis runs along the wall and the $y$ axis is directed into the liquid.

The boundary conditions (1) $j^{y}=0, w^{y}=0$, $j^{x}+\rho_{s} w^{x}=0$ can be written in the matrix form

where $\cos ^{2} \phi_{2}=1-\left(s_{2}^{2} / s^{2}\right) \sin ^{2} \phi_{1}$, to satisfy the condition $k_{I 1}^{x}=k_{R 2}^{x}$. The last term on the left-hand side of (18) represents the transverse surface wave with a wave vector $\mathbf{k}_{3}$. The wave must decay away from the boundary, therefore $\operatorname{Im} k_{3}^{y}>0$. This requirement selects the sign in (19), which is the transversality relation $\mathbf{w}_{3} \perp \mathbf{k}_{3}$ :

$$
\left(\begin{array}{c}
w_{3}^{x} \\
w_{3}^{y}
\end{array}\right) \propto\left(\begin{array}{c}
-k_{3}^{y} \\
k_{3}^{x}
\end{array}\right) \approx\left(\begin{array}{c}
-k_{3} \\
k_{1} \sin \phi_{1}
\end{array}\right)=\left(\begin{array}{c}
-\varkappa \mathrm{e}^{i \pi / 4} \\
k_{1} \sin \phi_{1}
\end{array}\right)=\left(\begin{array}{c}
-\varkappa \mathrm{e}^{i \pi / 4} \\
k_{2} \sin \phi_{2}
\end{array}\right)
$$

where $\varkappa=\sqrt{\omega \rho_{n} / \eta}$. Substituting this in (18) we get

$\left(\begin{array}{c}j_{I 1} \sin \phi_{1} \\ -j_{I 1} \cos \phi_{1} \\ 0\end{array}\right)+\left(\begin{array}{c}j_{R 1} \sin \phi_{1} \\ j_{R 1} \cos \phi_{1} \\ 0\end{array}\right)+\left(\begin{array}{c}\rho_{s} w_{R 2} \sin \phi_{2} \\ 0 \\ w_{R 2} \cos \phi_{2}\end{array}\right)+\left(\begin{array}{c}\rho w_{3}^{x} \\ \rho_{n} w_{3}^{y} \\ w_{3}^{y}\end{array}\right)=0$

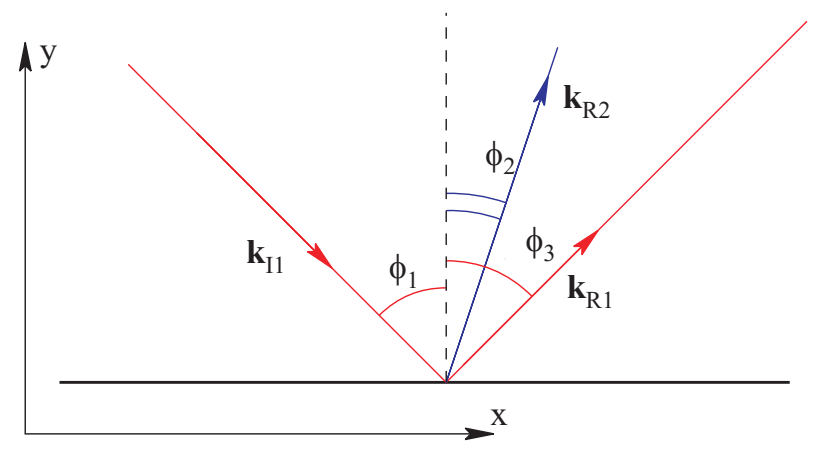

Fig. 1. First sound reflection. 
and

$$
2 j_{I 1}+w_{R 2}\left(\rho_{s} \frac{\sin \phi_{2}}{\sin \phi_{1}}+\rho_{n} \frac{\cos \phi_{2}}{\cos \phi_{1}}+\mathrm{e}^{i \pi / 4} \frac{\varkappa \rho \cos \phi_{2}}{k_{1} \sin ^{2} \phi_{1}}\right)=0 .
$$

Second sound is slower than first sound, i.e. $s>s_{2}$, consequently $\phi_{2}<\pi / 2$ and $\cos \phi_{2} \neq 0$. One can therefore neglect the first term in parenthesis

$$
w_{R 2}=-j_{I 1} \frac{\sin ^{2} \phi_{1}}{\cos \phi_{2}} \frac{2 k_{1}}{\rho_{n} k_{1} \sin \phi_{1} \tan \phi_{1}+\mathrm{e}^{i \pi / 4} \varkappa \rho} .
$$

Similarly, the amplitude of the reflected first sound is obtained from the equation

$$
\begin{gathered}
\left(\begin{array}{c}
j_{I 1} \rho_{n} k_{1} \sin ^{2} \phi_{1} \\
-j_{I 1} \rho \varkappa \mathrm{e}^{i \pi / 4} \cos \phi_{1}
\end{array}\right)+\left(\begin{array}{c}
j_{R 1} \rho_{n} k_{1} \sin ^{2} \phi_{1} \\
j_{R 1} \rho \varkappa \mathrm{e}^{i \pi / 4} \cos \phi_{1}
\end{array}\right)+ \\
+\left(\begin{array}{c}
\rho \rho_{n} k_{1} \sin \phi_{1} w_{3}^{x} \\
\rho \rho_{n} \varkappa \mathrm{e}^{i \pi / 4} w_{3}^{y}
\end{array}\right)=0 .
\end{gathered}
$$

From this we have

$$
j_{R 1}=j_{I 1} \frac{\rho \varkappa \cos \phi_{1}-\rho_{n} k_{1} \sin ^{2} \phi_{1} \mathrm{e}^{-i \pi / 4}}{\rho \varkappa \cos \phi_{1}+\rho_{n} k_{1} \sin ^{2} \phi_{1} \mathrm{e}^{-i \pi / 4}} .
$$

Reflection and conversion efficiency must be characterized by appropriate coefficients $R_{11}=F_{R 1} / F_{I 1}$ and $R_{12}=F_{R 2} / F_{I 1}$, respectively. Here $F_{1}$ and $F_{2}$ are the energy fluxes in the first and second sound waves. They are given by the expressions

$$
F_{1}=\frac{s}{2 \rho}|j|^{2}, F_{2}=\frac{s_{2} \rho_{s} \rho_{n}}{2 \rho}|w|^{2} .
$$

Using (21) and (20) we get

$$
\begin{gathered}
R_{11}=\left|\frac{\rho \varkappa \cos \phi_{1}-\rho_{n} k_{1} \sin ^{2} \phi_{1} \mathrm{e}^{-i \pi / 4}}{\rho \varkappa \cos \phi_{1}+\rho_{n} k_{1} \sin ^{2} \phi_{1} \mathrm{e}^{-i \pi / 4}}\right|^{2}, \\
R_{12}=\frac{s_{2}}{s} \frac{\sin ^{4} \phi_{1}}{\cos ^{2} \phi_{2}} \frac{4 \rho_{s} \rho_{n} k_{1}^{2}}{\left|\rho_{n} k_{1} \sin \phi_{1} \tan \phi_{1}+\rho \varkappa \mathrm{e}^{i \pi / 4}\right|^{2}} .
\end{gathered}
$$

Sample graph of these functions is illustrated on Fig. 2. The reflection coefficient $R_{11}$ has a minimum of

$$
\min R_{11}=3-2 \sqrt{2}
$$

at finite angle of incidence. The value at the minimum is the same as for the sound reflection in usual hydrodynamics [2].

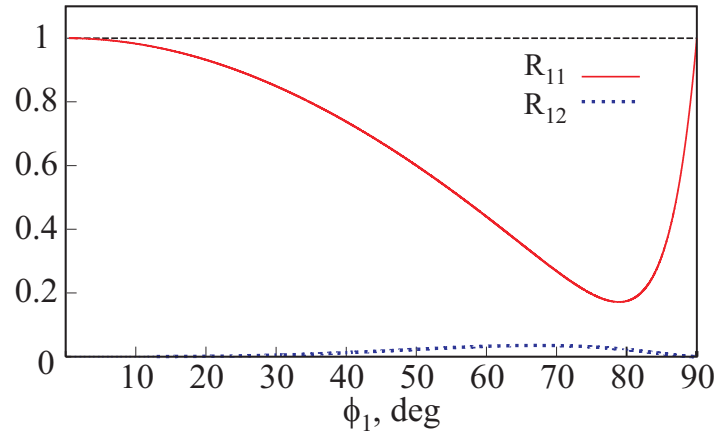

Fig. 2. Reflection and conversion coefficients $R_{11}$ and $R_{12}$ vs. the angle of incidence $\phi_{1}$.

\section{Second sound reflection}

The same approach can be used to investigate the second sound wave incident at an angle $\phi_{2}$ (see Fig. 3). The boundary conditions in this case are

$$
\begin{gathered}
\left(\begin{array}{c}
0 \\
0 \\
w_{I 2} \sin \phi_{2} \\
-w_{I 2} \cos \phi_{2}
\end{array}\right)+\left(\begin{array}{c}
0 \\
0 \\
w_{R 2} \sin \phi_{2} \\
w_{R 2} \cos \phi_{2}
\end{array}\right)+\left(\begin{array}{c}
j_{R 1} \sin \phi_{1} \\
j_{R 1} \cos \phi_{1} \\
0 \\
0
\end{array}\right)+ \\
+\left(\begin{array}{c}
\rho_{n} w_{3}^{x} \\
\rho_{n} w_{3}^{y} \\
w_{3}^{x} \\
w_{3}^{y}
\end{array}\right)=\left(\begin{array}{c}
-\rho_{s} w \\
0 \\
w \\
0
\end{array}\right) .
\end{gathered}
$$

After simplification this gives

$2 \rho_{s} \rho_{n} w_{I 2}=j_{R 1}\left(-\frac{\rho \varkappa \mathrm{e}^{i \pi / 4} \cos \phi_{1}}{k_{2} \sin ^{2} \phi_{2}}-\rho_{n} \frac{\sin \phi_{1}}{\sin \phi_{2}}-\rho_{s} \frac{\cos \phi_{1}}{\cos \phi_{2}}\right)$.

The last term in parenthesis is always negligible (the equation is meaningful only if $\sin \phi_{2}<s_{2} / s$ ). This gives

$$
j_{R 1}=-\rho_{s} w_{I 2} \frac{2 \rho_{n} k_{2} \sin ^{2} \phi_{2}}{\rho \varkappa \mathrm{e}^{i \pi / 4} \cos \phi_{1}+\rho_{n} k_{2} \sin \phi_{1} \sin \phi_{2}} .
$$

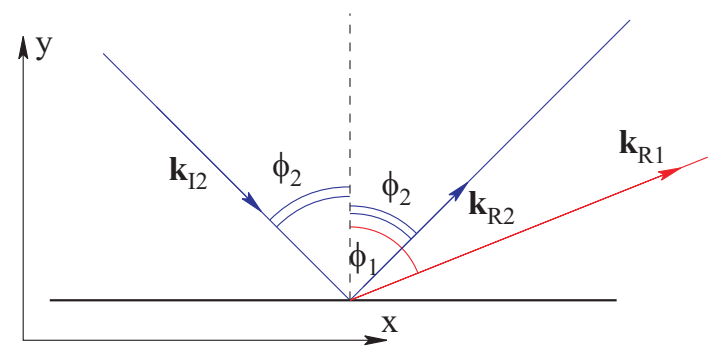

Fig. 3. Second sound reflection. 
The conversion coefficient $R_{21}=F_{R 1} / F_{I 2}$ is therefore given by

$$
R_{21}=\frac{4 s}{s_{2}} \frac{\rho_{s} \rho_{n} k_{2}^{2} \sin ^{4} \phi_{2}}{\left|\rho \varkappa \mathrm{e}^{i \pi / 4} \cos \phi_{1}+\rho_{n} k_{2} \sin \phi_{1} \sin \phi_{2}\right|^{2}} .
$$

Its maximum

$$
\max R_{21}=4 \frac{\rho_{s} s_{2}}{\rho_{n} s}
$$

is reached at the critical angle $\sin \phi_{2}=s_{2} / \mathrm{s}$.

Amplitude of the reflected second sound wave is found from the relation

$$
\begin{gathered}
\frac{w_{R 2}}{w_{I 2}}\left(\frac{\varkappa \rho \mathrm{e}^{i \pi / 4}}{k_{2} \sin \phi_{2}}+\rho_{n} \tan \phi_{1}+\rho_{s} \tan \phi_{2}\right)= \\
=\left(\frac{\varkappa \rho \mathrm{e}^{i \pi / 4}}{k_{2} \sin \phi_{2}}+\rho_{n} \tan \phi_{1}-\rho_{s} \tan \phi_{2}\right),
\end{gathered}
$$

where $\tan \phi_{1}=s \sin \phi_{2} / \sqrt{s_{2}^{2}-s^{2} \sin ^{2} \phi_{2}}$ and $\operatorname{Im}\left(\tan \phi_{1}\right) \leq 0$ (selected by the requirement $\operatorname{Im} k_{1}^{y} \geq 0$ ). The reflection coefficient is therefore

$$
R_{22}=\left|\frac{\rho \varkappa \mathrm{e}^{i \pi / 4}+\rho_{n} k_{2} \sin \phi_{2} \tan \phi_{1}-\rho_{s} k_{2} \sin \phi_{2} \tan \phi_{2}}{\rho \varkappa \mathrm{e}^{i \pi / 4}+\rho_{n} k_{2} \sin \phi_{2} \tan \phi_{1}+\rho_{s} k_{2} \sin \phi_{2} \tan \phi_{2}}\right|^{2} .
$$

These functions for sample parameters are plotted on Fig. 4.

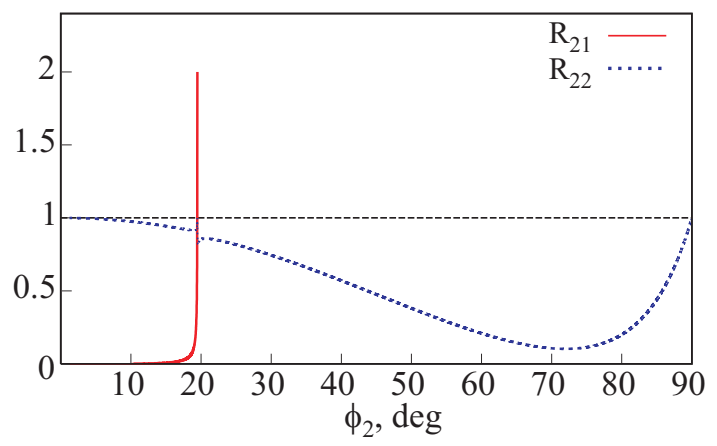

Fig. 4. Reflection and conversion coefficients $R_{22}$ and $R_{21}$ vs. the angle of incidence $\phi_{2}$. Note, that the energy conservation gives $R_{22} \cos \phi_{2}+R_{21} \cos \phi_{1} \leq \cos \phi_{2}$, and $R_{22}+R_{21}>1$ is not a violation!

\section{Discussion}

In helium II sound reflection at slanted incidence by a plane impervious wall is suppressed for both first and second sound. This phenomenon is similar to the Konstantinov effect in ordinary gases.

Coincident with the reflection suppression, a sound conversion takes place. The effect has strong angle dependence and should allow direct experimental verification. Moreover, there exist heat pulse propagation measurements [11] where the pulse transit time was often much shorter than that expected for second sound. This phenomenon is usually explained by an anomalously long phonon free path at low temperatures or by sound conversion in bulk (due to nonlinear effects) or at liquid-vapour interface. It seems probable that fast propagation is in fact a manifestation of the sound conversion described in this paper, so that the heat pulse is transformed at some wall into the pressure pulse and is later transformed back near the receiver. The signal therefore travels (some part of) the path with the velocity of first sound.

I thank A.F. Andreev and V.I. Marchenko for fruitful discussions. The work was supported in parts by RFBR grants 06-02-17369, 06-02-17281 and RF president program 7018.2006.2.

1. K.F. Herzfeld, Phys. Rev. 53 , 899 (1938).

2. B.P. Konstantinov, Zh. Tekh. Fiz. 9, 226 (1939).

3. L.D. Landau and E.M. Lifshitz, Fluid Mechanics, Pergamon Press, Oxford (1987).

4. L.D. Landau, J. Phys. USSR 5, 71 (1941).

5. K.R. Atkins, Phys. Rev. 113, 962 (1959).

6. J.R. Pellam, Phys. Rev. 73, 608 (1948).

7. R.B. Dingle, Proc. Phys. Soc. A61, 9 (1948).

8. D.M. Chernikova, Sov. Phys. JETP 20 , 358 (1965).

9. M.Yu. Kagan and Yu.A. Kosevich, Fiz. Nizk. Temp. 14, 787 (1988) [Sov. J. Low Temp. Phys. 14,433 (1988)].

10. I.M. Khalatnikov, An Introduction to the Theory of Superfluidity, W.A.Benjamin, New York-Amsterdam (1965).

11. J.R. Pellam, Phys. Rev. 75, 1183 (1949). 